\title{
Law Invariant Risk Measures have the Fatou Property *
}

\author{
E. Jouini ${ }^{\dagger} \quad$ W. Schachermayer ${ }^{\ddagger} \quad$ N. Touzi ${ }^{\S}$
}

\begin{abstract}
S. Kusuoka [K 01, Theorem 4] gave an interesting dual characterization of law invariant coherent risk measures, satisfying the Fatou property. The latter property was introduced by F. Delbaen [D 02]. In the present note we extend Kusuoka's characterization in two directions, the first one being rather standard, while the second one is somewhat surprising. Firstly we generalize - similarly as M. Fritelli and E. Rossaza Gianin [FG 05] - from the notion of coherent risk measures to the more general notion of convex risk measures as introduced by H. Föllmer and A. Schied [FS 04]. Secondly - and more importantly - we show that the hypothesis of Fatou property may actually be dropped as it is automatically implied by the hypothesis of law invariance.

We also introduce the notion of the Lebesgue property of a convex risk measure, where the inequality in the definition of the Fatou property is replaced by an equality, and give some dual characterizations of this property.
\end{abstract}

\section{Introduction}

This paper is a twin to [JST 05] and we shall use similar notation. In particular we rather use the language of "monetary utility functions" which — up to the

${ }^{*}$ We thank S. Kusuoka, P. Orihuela and A. Schied for their advise and help in preparing this paper.

†Université Paris Dauphine and CEREMADE, Place du Maréchal de Lattre de Tassigny, F-75775 Paris Cedex 16, France.

$\ddagger$ Vienna University of Technology, Wiedner Hauptstrasse 8-10/105, A-1040 Wien, Austria and Université Paris Dauphine, Place du Maréchal de Lattre de Tassigny, F-75775 Paris Cedex 16, France. Financial support from the Austrian Science Fund (FWF) under the grant P15889 and from Vienna Science and Technology Fund (WWTF) under Grant MA13 is gratefully acknowledged.

${ }^{\S}$ CREST, Laboratoire de Finance et Assurance, 15 Bd Gabriel Péri, F-92245 Malakoff Cedex, France and Université Paris Dauphine, Place du Maréchal de Lattre de Tassigny, F-75775 Paris Cedex 16, France. 
sign - is identical to the notion of convex risk measures [FS 04]. We do so in order to point out more directly how the present theory is embedded into the framework of classical utility theory.

Throughout the paper we work on a standard probability space $(\Omega, \mathcal{F}, \mathbf{P})$, i.e., we suppose that $(\Omega, \mathcal{F}, \mathbf{P})$ does not have atoms and that $\mathbb{L}^{2}(\Omega, \mathcal{F}, \mathbf{P})$ is separable.

A monetary utility function is a concave non-decreasing map $U$ : $\mathbb{L}^{\infty}(\Omega, \mathcal{F}, \mathbf{P}) \rightarrow[-\infty, \infty[$ with $\operatorname{dom}(U)=\{X \mid U(X) \in \mathbb{R}\} \neq \emptyset$, and

$$
U(X+c)=U(X)+c, \text { for } X \in \mathbb{L}^{\infty}, c \in \mathbb{R} .
$$

Note that a monetary utility function is Lipschitz with respect to $\|\cdot\|_{\infty}$, and that $\operatorname{dom}(U)=\mathbb{L}^{\infty}$. By adding a constant to $U$ if necessary, we may and shall always assume that $U(0)=0$.

Defining $\rho(X)=-U(X)$ the above definition of a monetary utility function yields the definition of a convex risk measure [FS 04]. Convex risk measures are in turn a generalization of the concept of coherent risk measures [ADEH 97], which are particularly relevant in applications, and where one imposes the additional requirement of positive homogeneity $\rho(\lambda X)=\lambda \rho(X)$, for $X \in \mathbb{L}^{\infty}$ and $\lambda \geq 0$. A characterization of coherent (resp. convex) risk measures $\rho$ : $\mathbb{L}^{\infty}(\Omega, \mathcal{F}, \mathbf{P}) \rightarrow \mathbb{R}$ in terms of their Fenchel transform, defined on $\mathbb{L}^{1}(\Omega, \mathcal{F}, \mathbf{P})$, was obtained in [D 02] under the condition that $\rho$ satisfies the Fatou property, i.e.,

$$
\rho(X) \leq \liminf _{n \rightarrow \infty} \rho\left(X_{n}\right) \quad \text { whenever } \sup _{n}\left\|X_{n}\right\|_{\infty}<\infty \text { and } X_{n} \stackrel{\mathbf{P}}{\longrightarrow} X,
$$

where $\stackrel{\mathbf{P}}{\longrightarrow}$ denotes convergence in probability. In the present context, this condition is equivalent to the upper semi-continuity condition with respect to the $\sigma\left(\mathbb{L}^{\infty}, \mathbb{L}^{1}\right)$-topology.

For fixed $X \in \mathbb{L}^{1}(\Omega, \mathcal{F}, \mathbf{P})$, we introduce the function

$$
\left.U_{\alpha}(X):=\alpha^{-1} \int_{0}^{\alpha} q_{X}(\beta) d \beta, \quad \alpha \in\right] 0,1[,
$$

$U_{0}(X)=\operatorname{ess} \inf (X)$, and $U_{1}(X)=\mathbf{E}[X]$, where $q_{X}$ denotes the quantile function of the random variable $X$, i.e. the generalized inverse of its cumulative distribution function (see (3) below). For every $\alpha \in[0,1], U_{\alpha}$ is a positively homogeneous monetary utility function, which is in addition law invariant. The corresponding coherent risk measure $\rho_{\alpha}=-U_{\alpha}$ is the so-called average value at risk at level $\alpha$, sometimes denoted by $\mathrm{AV} @ \mathrm{R}_{\alpha}$ (see [FS 04]). The family $\left\{U_{\alpha}, 0 \leq \alpha \leq 1\right\}$ plays an important role as any law invariant monetary utility function $U$ may be represented in terms of the utility functions $U_{\alpha}$, $\alpha \in[0,1]$. This result was obtained by [K01] in the context of coherent risk measures, and later extended by [FG05] to the context of convex risk measures, see also [FS 04], Theorem 4.54 and 4.57 as well as Corollary 4.72. The precise statement of this result is the following. 
Theorem 1.1 Suppose that $(\Omega, \mathcal{F}, \mathbf{P})$ is a standard probability space. For a function $U: \mathbb{L}^{\infty}(\Omega, \mathcal{F}, \mathbf{P}) \rightarrow \mathbb{R}$ the following are equivalent:

(a) $U$ is a law invariant monetary utility function satisfying the Fatou property.

(b) There is a convex function $v: \mathcal{P}([0,1]) \rightarrow[0, \infty]$ such that

$$
U(X)=\inf _{m \in \mathcal{P}([0,1])}\left\{\int_{0}^{1} U_{\alpha}(X) d m(\alpha)+v(m)\right\} \text { for every } X \in \mathbb{L}^{\infty} .
$$

Here, $\mathcal{P}([0,1])$ denotes the set of all Borel probability measures on the compact space $[0,1]$. The crucial observation of Kusuoka [K 01] is that, for law invariant monetary utility functions, condition (b) is equivalent to

(c) There is a law invariant, lower semi-continuous, convex function $V$ : $\mathbb{L}^{1}(\Omega, \mathcal{F}, \mathbf{P}) \rightarrow[0, \infty]$ such that $\operatorname{dom}(V) \subseteq \mathcal{P}(\Omega, \mathcal{F}, \mathbf{P})$ and

$$
U(X)=\inf _{Y \in \mathbb{L}^{1}}\{\mathbf{E}[X Y]+V(Y)\} \quad \text { for every } X \in \mathbb{L}^{\infty},
$$

where $\mathcal{P}(\Omega, \mathcal{F}, \mathbf{P})$ denotes the set of $\mathbf{P}$-absolutely continuous probability measures on $(\Omega, \mathcal{F}, \mathbf{P})$, which we identify with a subset of $\mathbb{L}^{1}(\Omega, \mathcal{F}, \mathbf{P})$. For completeness, we report a proof of the equivalence between conditions (b) and (c) in Section 3.

The equivalence of (a) and (c) is due to F. Delbaen in the framework of (not necessarily law invariant) coherent risk measures [D 02], and was extended to convex risk measures in [FS 04].

The first main contribution of this paper is to drop the Fatou property in condition (a) of the above Theorem 1.1 by proving that it is automatically satisfied by law-invariant monetary utility functions. In fact, we prove more generally that the Fatou property is implied by the concavity, the $\mathbb{L}^{\infty}$-u.s.c. and the law-invariance properties. This result is stated in Section 2 and proved in Section 4. The reader only interested in this result may directly proceed to these sections.

We next introduce the following natural notion.

Definition 1.2 A utility function $U: \mathbb{L}^{\infty} \rightarrow \mathbb{R} \cup\{-\infty\}$ satisfies the Lebesgue property if for every uniformly bounded sequence $\left(X_{n}\right)_{n=1}^{\infty}$ tending a.s. to $X$ we have

$$
U(X)=\lim _{n \rightarrow \infty} U\left(X_{n}\right)
$$

Clearly the Lebesgue property is a stronger condition than the Fatou property defined in (1), as the inequality has been replaced by an equality. In fact, this property was - under different names - already investigated in the previous literature, as was kindly pointed out to us by A. Schied. 
The second contribution of this paper is a characterization of the Lebesgue property for a monetary utility function $U$ in terms of the corresponding Fenchel transform $V$ introduced in condition (c) of Theorem 1.1. If in addition $U$ is law-invariant, this implies a characterization in terms of the function $v$ introduced in the above Theorem 1.1(b). These results are stated in Section 2 and proved in Section 5.

\section{AV@R representation of law-invariant mon- etary utilities}

\subsection{Definitions}

Let $(\Omega, \mathcal{F}, \mathbf{P})$ be an atomless probability space, and assume that $\mathbb{L}^{2}(\Omega, \mathcal{F}, \mathbf{P})$ is separable. The assumption of $\mathcal{F}$ being free of atoms is crucial (otherwise one is led to combinatorial problems which are irrelevant from the economic point of view). On the other hand, the separability assumption is convenient for the arguments below, but does not reduce the generality: indeed in all the arguments below we shall only encounter (at most) countably many random variables $\left(X_{n}\right)_{n=1}^{\infty}$; hence we may assume w.l.g. that the $\sigma$-algebra $\mathcal{F}$ is generated by countably many random variables, i.e., that $\mathbb{L}^{2}(\Omega, \mathcal{F}, \mathbf{P})$ is separable.

We denote by $\mathcal{P}(\Omega, \mathcal{F}, \mathbf{P})$ the set of $\mathbf{P}$-absolutely continuous probability measures on $(\Omega, \mathcal{F}, \mathbf{P})$, which we identify with a subset of $\mathbb{L}^{1}(\Omega, \mathcal{F}, \mathbf{P})$. We also denote by $\mathcal{P}([0,1])$ (resp. $\mathcal{P}(] 0,1])$ ) the set of all Borel probability measures on the compact space $[0,1]$ (resp. on the locally compact space $] 0,1]$ ).

A measure preserving transformation of $(\Omega, \mathcal{F}, \mathbf{P})$ is a bi-measurable bijection $\tau: \Omega \rightarrow \Omega$ leaving $\mathbf{P}$ invariant, i.e., $\tau(\mathbf{P})=\mathbf{P}$. For $1 \leq p \leq \infty$, the transformation $\tau$ induces an isometric isomorphism, still denoted by $\tau$, on $\mathbb{L}^{p}(\Omega, \mathcal{F}, \mathbf{P})$, mapping $X$ to $X \circ \tau$.

A map $f: \mathbb{L}^{\infty} \rightarrow \mathbb{R}$ is called law invariant, if $f(X)$ depends only on the law of $X$ for every $X \in \mathbb{L}^{\infty}$. The function $f$ is called transformation invariant if $f \circ \tau=f$ for every measure preserving transformation $\tau$, where we abuse notations by writing $f \circ \tau(X):=f(X \circ \tau)$.

We shall verify in Lemma A.4 that these notions of law invariance and transformation invariance may be used in a synonymous way in the present context of monetary utility function, as a consequence of the concavity and the $\|\cdot\|_{\infty}$-continuity property of monetary utility functions.

An important example of law invariant and transformation invariant function is the so-called quantile function defined by

$$
q_{X}(\alpha):=\inf \{x \in \mathbb{R} \mid \mathbf{P}[X \leq x] \geq \alpha\}, \quad X \in \mathbb{L}^{\infty}, \alpha \in[0,1] .
$$

The functions $U_{\alpha}, 0 \leq \alpha \leq 1$, introduced in (2) provide the simplest example of law invariant monetary utility functions, which correspond to the so-called 
average value at risk.

\subsection{Strong and weak upper semi-continuity of law in- variant maps}

We now have assembled all the concepts that are needed to formulate our first main result.

Theorem 2.1 Suppose that $(\Omega, \mathcal{F}, \mathbf{P})$ is a standard probability space. For a function $U: \mathbb{L}^{\infty}(\Omega, \mathcal{F}, \mathbf{P}) \rightarrow \mathbb{R}$ the following are equivalent:

(i) $U$ is a law invariant monetary utility function.

(ii) There is a law invariant, lower semi-continuous, convex function $V$ : $\mathbb{L}^{1}(\Omega, \mathcal{F}, \mathbf{P}) \rightarrow[0, \infty]$ such that $\operatorname{dom}(V) \subseteq \mathcal{P}(\Omega, \mathcal{F}, \mathbf{P})$ and

$$
U(X)=\inf _{Y \in \mathbb{L}^{1}}\{\mathbf{E}[X Y]+V(Y)\} \quad \text { for } \quad X \in \mathbb{L}^{\infty} .
$$

(iii) There is a convex function $v: \mathcal{P}([0,1]) \rightarrow[0, \infty]$ such that

$$
U(X)=\inf _{m \in \mathcal{P}([0,1])}\left\{\int_{0}^{1} U_{\alpha}(X) d m(\alpha)+v(m)\right\} \text { for } X \in \mathbb{L}^{\infty} .
$$

If any of these conditions is satisfied, then $U$ satisfies the Fatou property.

This result shows that law invariant monetary utility functions admit a representation in terms of the corresponding Fenchel transform without any further assumption. In particular the AV@R representation of such utility functions holds without any further condition. Our novel contribution is that the Fatou property is automatically implied by the law invariance and the strong upper semi-continuity; recall that monetary utility functions are $\mathbb{L}^{\infty}$ Lipschitz continuous. We state this fact in a slightly more general framework:

Theorem 2.2 Suppose that $(\Omega, \mathcal{F}, \mathbf{P})$ is a standard probability space. Let $U$ : $\mathbb{L}^{\infty} \rightarrow \mathbb{R} \cup\{-\infty\}$ be a concave function, which is law invariant and u.s.c. with respect to the topology induced by \|\|$_{\infty}$. Then $U$ is u.s.c. with respect to the $\sigma\left(\mathbb{L}^{\infty}, \mathbb{L}^{1}\right)$-topology.

We prove this result, which we consider as the main contribution of this paper, in section 4 . The reader only interested in Theorem 2.2 may directly proceed to this section.

Finally we observe that Theorem 2.1 implies in particular that in Theorem 7 of [K 01] the assumption of the Fatou property may also be dropped. For the sake of completeness we formulate this result.

A monetary utility function $U: \mathbb{L}^{\infty} \rightarrow \mathbb{R}$ is called comonotone if $U\left(X_{1}+\right.$ $\left.X_{2}\right)=U\left(X_{1}\right)+U\left(X_{2}\right)$, for comonotone $X_{1}, X_{2} \in \mathbb{L}^{\infty}$ (compare [JST 05]). Note that this implies in particular that $U$ is positively homogeneous, i.e., 
$U(\lambda X)=\lambda U(X)$, for $\lambda \geq 0$, so that $\rho(X):=-U(X)$ is a coherent risk measure.

Theorem 2.3 Suppose that $(\Omega, \mathcal{F}, \mathbf{P})$ is a standard probability space. For a function $U: \mathbb{L}^{\infty}(\Omega, \mathcal{F}, \mathbf{P}) \rightarrow \mathbb{R}$ the following are equivalent:

(i) $U$ is a comonotone, law invariant, monetary utility function.

(ii) There is a probability measure $m$ on $[0,1]$ such that

$$
U(X)=\int_{0}^{1} U_{\alpha}(X) d m(\alpha), \quad X \in \mathbb{L}^{\infty} .
$$

In fact, this latter result is known, and may be found in [FS 04], Theorem 4.87, as was kindly pointed out to us by A. Schied.

\subsection{Dual characterization of the Lebesgue property}

Under the additional assumption of $U$ satisfying the Lebesgue property of Definition 1.2, we have the following variant of Theorem 2.1.

Theorem 2.4 For a function $U: \mathbb{L}^{\infty}(\Omega, \mathcal{F}, \mathbf{P}) \rightarrow \mathbb{R}$ the following are equivalent:

(i) $U$ is a law invariant monetary utility function satisfying the Lebesgue property.

(ii) There is a law invariant, lower semi-continuous, convex function $V$ : $\mathbb{L}^{1}(\Omega, \mathcal{F}, \mathbf{P}) \rightarrow[0, \infty]$ such that $\operatorname{dom}(V) \subseteq \mathcal{P}(\Omega, \mathcal{F}, \mathbf{P})$,

$$
U(X)=\inf _{Y \in \mathbb{L}^{1}}\{\mathbf{E}[X Y]+V(Y)\}, \quad X \in \mathbb{L}^{\infty},
$$

and $\{V \leq c\}$ is uniformly integrable, for each $c>0$.

(iii) There is a convex function $v: \mathcal{P}(] 0,1]) \rightarrow[0, \infty]$ such that

$$
U(X)=\inf _{m \in \mathcal{P}(] 0,1])}\left\{\int_{0}^{1} U_{\alpha}(X) d m(\alpha)+v(m)\right\}, \quad X \in \mathbb{L}^{\infty},
$$

and such that, for $c>0,\{v \leq c\}$ is relatively compact in the Prokhorov topology on $\mathcal{P}(] 0,1])$, i.e., for $c>0$ and $\varepsilon>0$ there exists $\alpha>0$ such that $m(] 0, \alpha])<\varepsilon$, whenever $v(m) \leq c$.

To relate Theorem 2.4 to Theorem 2.1 it is instructive to consider a very easy example, namely $U_{0}(X)=\operatorname{ess} \inf (X)$, which is a law invariant monetary utility function. It is straight-forward to verify that $U_{0}$ fails the Lebesgue property. In this case the functions $V$ (resp. $v$ ) appearing in (ii) and (iii) of Theorem 2.1 simply are identically zero on $\mathcal{P}(\Omega, \mathcal{F}, \mathbf{P})$ (resp. on $\mathcal{P}(] 0,1])$ ), so that they do not satisfy the uniform integrability (resp. relative compactness) requirements in (ii) and (iii) of Theorem 2.1. 
We also stress the difference of $\mathcal{P}([0,1])$ versus $\mathcal{P}(] 0,1])$ in Theorem 2.1 and 2.4 respectively. The above formulation of Theorem 2.1 using $\mathcal{P}([0,1])$ was stated by S. Kusuoka and seems more natural (although it would be possible to also formulate Theorem 2.1 using $\mathcal{P}(] 0,1])$ instead of $\mathcal{P}([0,1])$. For the formulation of Theorem 2.3, however, it is indispensable to pass to $\mathcal{P}([0,1]))$. Think again of $U^{0}(X)=\operatorname{ess} \inf (X)$. In this case, the measure $m$ appearing in Theorem 2.3 (ii) equals the Dirac-measure $\delta_{0}$.

\section{Reduction of the probability space by law invariance}

In this section we shall show the equivalence of (ii) and (iii) in Theorem 2.1. We shall see that a rather straight-forward application of the formula of integration by parts translates (ii) into (iii) and vice versa. To do so rigourously, it will be convenient to develop some functional analytic machinery.

As in [JST 05] we denote by $\mathcal{D} \searrow$ the set of non-increasing, right continuous, $\mathbb{R}_{+}$-valued functions $f$ on $\left.] 0,1\right]$ such that $f(1)=0$ and

$$
\|f\|_{1}=\int_{0}^{1} f(x) d x=1 .
$$

We define the map $T: \mathcal{D} \backslash \rightarrow \mathcal{M}(] 0,1])$ by $T(f)=m$ where the measure $m$ on the locally compact space $] 0,1]$ is defined by

$$
d m(x)=-x d f(x), \quad x \in] 0,1] .
$$

To verify that (6) well-defines a probability measure on ]0,1] suppose first that $f$ is differentiable and bounded on $] 0,1]$. We then may apply the classical formula of integration by parts to obtain

$$
\begin{aligned}
\left.\left.\|m\|_{1}=m(] 0,1\right]\right) & =\int_{0}^{1} d m(x) \\
& =-\int_{0}^{1} x f^{\prime}(x) d x \\
& =-[x f(x)]_{0}^{1}+\int_{0}^{1} f(x) d x=\|f\|_{1}=1 .
\end{aligned}
$$

This isometric identity also remains valid for arbitrary $f \in \mathcal{D} \backslash$ : indeed, by considering $f \wedge c$, for $c>0$ renormalizing and letting $c \rightarrow \infty$, one reduces to the case of bounded $f$; for general bounded $f \in \mathcal{D} \searrow$ it suffices to interpret the above partial integration formula in a generalized sense, using Stieltjes integration.

In fact, the map $T: \mathcal{D} \searrow \rightarrow \mathcal{M}(] 0,1])$ defines a bijection between $\mathcal{D} \searrow$ and the set $\mathcal{P}([0,1])$ of probability measures on the locally compact space $] 0,1]$. 
Indeed, one may interpret (6) just as well as a definition of the function $f$ (right-continuous and satisfying $f(1)=0$ ) for given $m \in \mathcal{P}(10,1])$.

We still observe that $T$ maps, for $\alpha \in] 0,1]$, the functions $g_{\alpha}=\alpha^{-1} \mathbf{1}_{] 0, \alpha[} \in$ $\mathcal{D} \backslash$ to the Dirac measure $\delta_{\alpha}$ on $\left.] 0,1\right]$; this property could just as well have been used to define the map $T$ (extending subsequently the definition by linearity and continuity).

Proof of Theorem 2.1 (ii) $\Leftrightarrow$ (iii)

Step 1: Given a convex function $v: \mathcal{P}(] 0,1]) \rightarrow[0, \infty]$ as in (iii) we define the function $V: \mathbb{L}^{1}(\Omega, \mathcal{F}, \mathbf{P}) \rightarrow[0, \infty]$ by

$$
V(Y)=v\left(T\left(-q_{-Y}\right)\right), Y \in \mathcal{P}(\Omega, \mathcal{F}, \mathbf{P}) \text { and } V(Y)=+\infty \text { otherwise, }(8)
$$

where $T$ is defined in (6). This is a convex law invariant function on $\mathbb{L}^{1}$.

Conversely, given a law invariant convex function $V: \mathbb{L}^{1}(\Omega, \mathcal{F}, \mathbf{P}) \rightarrow[0, \infty]$ we may well-define $\mathcal{V}: \mathcal{D} \searrow \rightarrow[0, \infty]$ by

$$
\mathcal{V}\left(-q_{-Y}\right):=V(Y)
$$

where $-q_{-Y}$ runs through $\mathcal{D} \searrow$ when $Y$ ranges through $\mathcal{P}(\Omega, \mathcal{F}, \mathbf{P})$. We then define $v: \mathcal{P}(] 0,1]) \rightarrow[0, \infty]$ by

$$
\left.\left.v(m)=\mathcal{V}\left(T^{-1}(m)\right), \quad m \in \mathcal{P}(] 0,1\right]\right)
$$

This establishes a bijective correspondence between the functions $V$ and $v$ as appearing in items (ii) and (iii) of Theorem 2.4. We have to show that two such functions $V$ and $v$ define the same function $U: \mathbb{L}^{\infty} \rightarrow \mathbb{R}$ via (4) and (5) respectively.

So fix $v$ and $V$ satisfying (10). Write $U^{V}$ for the function defined by (4) and $U^{v}$ for the function defined by (5).

First note that

$$
\begin{aligned}
U^{V}(X) & =\inf _{Y \in \mathbb{L}^{1}}\left\{\int_{0}^{1} q_{X}(\alpha)\left(-q_{-Y}(\alpha)\right) d \alpha-V(Y)\right\} \\
& =\inf _{f \in \mathcal{D}_{\searrow}}\left\{\int_{0}^{1} q_{X}(\alpha) f(\alpha) d \alpha-\mathcal{V}(f)\right\}
\end{aligned}
$$

Indeed, looking at the right hand side of (4), the term $V(Y)$ is invariant, when $Y$ runs through all elements of $\mathbb{L}^{1}(\Omega, \mathcal{F}, \mathbf{P})$ with fixed quantile function $-q_{-Y}(.) \in \mathcal{D} \searrow$. On the other hand the term $\mathbf{E}[X Y]$ becomes minimal, for fixed $X$ and law of $Y$, if $X$ and $Y$ are anti-comonotonic (compare [JST 05]), in which case

$$
\mathbf{E}[X Y]=\int_{0}^{1} q_{X}(\alpha)\left(-q_{-Y}(\alpha)\right) d \alpha
$$


which readily shows (11). Suppose now that $f(\alpha)=-q_{-Y}(\alpha)$ is bounded and differentiable on $] 0,1]$ to again apply integration by parts. Let $F(\alpha)=$ $\int_{0}^{\alpha} q_{X}(\beta) d \beta$ so that $U_{\alpha}(X)=\alpha^{-1} F(\alpha)$, for $\left.\left.\alpha \in\right] 0,1\right]$. We then have

$$
\begin{aligned}
\int_{0}^{1} q_{X}(\alpha) f(\alpha) d \alpha & =[F(\alpha) f(\alpha)]_{0}^{1}-\int_{0}^{1} F(\alpha) f^{\prime}(\alpha) d \alpha \\
& =-\int_{0}^{1} U_{\alpha}(X) \alpha f^{\prime}(\alpha) d \alpha \\
& =\int_{0}^{1} U_{\alpha}(X) d m(\alpha) .
\end{aligned}
$$

Note that the latter integral is just the term appearing in (5). Similarly as in (7) one verifies that (12) in fact holds true for arbitrary $f \in \mathcal{D} \searrow$, which readily shows that the functions $U^{V}$ and $U^{v}$ defined by (4) and (5) respectively coincide.

We still have to verify that the function $V$ in Theorem 2.1 (ii) may be assumed to be lower semi-continuous with respect to $\|\cdot\|_{1}$. In fact, this is a triviality: we may always pass from a law invariant, convex function $\widetilde{V}$ : $\mathbb{L}^{1} \rightarrow[0, \infty]$ to its lower semi-continuous envelope $V$, i.e., the largest lower semi-continuous function dominated by $V$. It now suffices to note that the passage from $\widetilde{V}$ to $V$ does not affect the conjugate function $U$ defined in (1), in other words $U^{V}(X)=U^{\widetilde{V}}(X)$.

Step 2: We now show that we may choose the function $v$ in (iii) to be defined

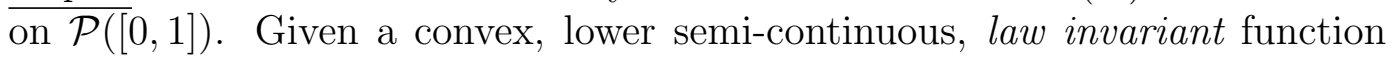
$V: \mathbb{L}^{1} \rightarrow[0, \infty]$ as in (ii), define the corresponding function $\left.\left.v: \mathcal{P}(] 0,1\right]\right) \rightarrow$ $[0, \infty]$ as in the above step 1 . We define the lower semi-continuous envelope $\bar{v}$ of $v$ on $\mathcal{P}([0,1])$ by

$$
\left.\left.\bar{v}(m):=\inf \left\{\lim _{n \rightarrow \infty} v\left(m_{n}\right) \mid\left(m_{n}\right)_{n=1}^{\infty} \in \mathcal{P}(] 0,1\right]\right) \text { and } \lim _{n \rightarrow \infty} m_{n}=m\right\},
$$

where the limit is taken with respect to the weak topology on $\mathcal{P}([0,1])$, i.e., the one generated by the continuous functions on $[0,1]$. Noting that, for $X \in$ $\mathbb{L}^{\infty}(\Omega, \mathcal{F}, \mathbf{P})$, the function $\alpha \mapsto U_{\alpha}(X)$ is continuous on $[0,1]$, we get

$$
\begin{aligned}
& \inf _{m \in \mathcal{P}(] 0,1])}\left\{\int_{0}^{1} U^{\alpha}(X) d m(\alpha)+v(m)\right\} \\
& =\inf _{m \in \mathcal{P}([0,1])}\left\{\int_{0}^{1} U^{\alpha}(X) d m(\alpha)+\bar{v}(m)\right\} .
\end{aligned}
$$

Hence we have found a (lower semi-continuous, convex) function $\bar{v}$, defined on $\mathcal{P}([0,1])$, such that the above infima coincide. We note in passing that, if $v$ satisfies the Prokhorov condition of (iii) of Theorem 2.4, then $\bar{v}(m)=\infty$, whenever $m(\{0\})>0$. 
Conversely starting with a function $v: \mathcal{P}([0,1]) \rightarrow[0, \infty]$, which we assume w.l.g. convex and l.s.c. by passing to this envelope, we may associate a convex, l.s.c., law invariant function $V: \mathbb{L}^{1}(\Omega, \mathcal{F}, \mathbf{P}) \rightarrow[0, \infty]$ as in the above step 1 . Note that the definition of $V$ only uses the restriction of $v$ to $\mathcal{P}(] 0,1])$. By (13) and the arguments in step 1, we conclude again that

$$
\begin{aligned}
& \inf _{m \in \mathcal{P}([0,1])}\left\{\int_{0}^{1} U^{\alpha}(X) d m(\alpha)+v(m)\right\} \\
& =\inf _{Y \in \mathbb{L}^{1}}\{\mathbf{E}[X Y]+V(Y)\} .
\end{aligned}
$$

This finishes the proof of Theorem 2.1.

We observe that, in the above proof of Theorem 2.1 (ii) $\Leftrightarrow$ (iii), we have in fact translated statement (iii) of Theorem 2.1 into the subsequent equivalent form:

(iii') There is a convex function $\mathcal{V}: \mathcal{D} \searrow \rightarrow[0, \infty]$ such that

$$
U(X)=\inf _{f \in \mathcal{D} \searrow}\left\{\int_{0}^{1} q_{X}(\alpha) f(\alpha) d \alpha-\mathcal{V}(f)\right\}
$$

Indeed, the translation of the law invariant function $V$ on $\mathbb{L}^{1}$ into the function $\mathcal{V}$ on $\mathcal{D} \searrow$ was done in (9) above and in (11) it was shown that the function $U(X)$ in (14) indeed equals the function $U^{V}(X)$.

We also remark that the above proof also shows that in item (ii) above one may equivalently drop the word "convex" and/or the word "lower semicontinuous".

We have imposed in item (ii) the condition of lower semi-continuity of the function $V$ in order to make sure that the terms "law invariant" and "transformation invariant" are equivalent (see Lemma A.4 below).

\section{The Fatou property for law invariant utility functions}

In this section we shall prove Theorem 2.2 which will follow from the subsequent result whose proof will be reported at the end of this section.

Proposition 4.1 Let $C$ be a convex, $\sigma^{*}$-closed, law invariant subset of $\mathbb{L}^{\infty}(\Omega, \mathcal{F}, \mathbf{P})^{*}$. Then $C \cap \mathbb{L}^{1}(\Omega, \mathcal{F}, \mathbf{P})$ is $\sigma^{*}$-dense in $C$. 
Hence for a law invariant convex $\sigma^{*}$-lower semi-continuous function $V: \mathbb{L}^{\infty}(\Omega, \mathcal{F}, \mathbf{P})^{*} \rightarrow[0, \infty], V$ equals the $\sigma^{*}$-lower semi-continuous extension of the restriction of $V$ to $\mathbb{L}^{1}(\Omega, \mathcal{F}, \mathbf{P})$, i.e.

$$
V(\mu)=\inf \left\{\lim _{\alpha \in I} V\left(f_{\alpha}\right) \mid\left(f_{\alpha}\right)_{\alpha \in I} \in \mathbb{L}^{1}, \sigma^{*}-\lim _{\alpha \in I} f_{\alpha}=\mu\right\}, \quad \mu \in\left(\mathbb{L}^{\infty}\right)^{*} .
$$

Some explanation seems in order. On $\left(\mathbb{L}^{\infty}\right)^{*}$ we consider the $\sigma^{*}=\sigma\left(\left(\mathbb{L}^{\infty}\right)^{*}, \mathbb{L}^{\infty}\right)$ topology and identify $\mathbb{L}^{1}(\Omega, \mathcal{F}, \mathbf{P})$ with a subspace of $\mathbb{L}^{\infty}(\Omega, \mathcal{F}, \mathbf{P})^{*}$. A measure preserving transformation $\tau:(\Omega, \mathcal{F}, \mathbf{P}) \rightarrow(\Omega, \mathcal{F}, \mathbf{P})$ defines an isometry, denoted again by $\tau$, on $\mathbb{L}^{p}(\Omega, \mathcal{F}, \mathbf{P})$, for $1 \leq p \leq \infty$, via

$$
\begin{aligned}
\tau: \mathbb{L}^{p} & \rightarrow \mathbb{L}^{p} \\
f & \mapsto f \circ \tau .
\end{aligned}
$$

The transpose of $\tau: \mathbb{L}^{\infty} \rightarrow \mathbb{L}^{\infty}$, denoted by $\tau^{*}$, defines an isometry on $\left(\mathbb{L}^{\infty}\right)^{*}$ via

$$
\begin{aligned}
\tau^{*}:\left(\mathbb{L}^{\infty}\right)^{*} & \rightarrow\left(\mathbb{L}^{\infty}\right)^{*} \\
\left\langle\tau^{*}(\mu), f\right\rangle & =\langle\mu, \tau(f)\rangle, \quad \mu \in\left(\mathbb{L}^{\infty}\right)^{*}, f \in \mathbb{L}^{\infty} .
\end{aligned}
$$

A function $V$ on $\left(\mathbb{L}^{\infty}\right)^{*}$ is called transformation invariant if $V=V \circ \tau^{*}$ for every measure preserving transformation $\tau:(\Omega, \mathcal{F}, \mathbf{P}) \rightarrow(\Omega, \mathcal{F}, \mathbf{P})$. A similar definition applies to subsets of $\left(\mathbb{L}^{\infty}\right)^{*}$.

A $\sigma^{*}$-closed convex subset $C$ of $\left(\mathbb{L}^{\infty}\right)^{*}$ is called law invariant if, for $X_{1}, X_{2} \in$ $\mathbb{L}^{\infty}$ with law $\left(X_{1}\right)=\operatorname{law}\left(X_{2}\right)$ we have

$$
\left\{\left\langle\mu, X_{1}\right\rangle \mid \mu \in C\right\}=\left\{\left\langle\mu, X_{2}\right\rangle \mid \mu \in C\right\} .
$$

A convex $\sigma^{*}$-lower semi-continuous function $V:\left(\mathbb{L}^{\infty}\right)^{*} \rightarrow \mathbb{R} \cup\{+\infty\}$ is called law invariant if, for each $c \in \mathbb{R}$, the level set $\{V \leq c\}$ is law invariant.

In Lemma A.5 below we justify that in our setting we may use the notions of law invariance and transformation invariance synonymously.

Admitting the above Proposition 4.1 the proof of Theorem 2.2 is straightforward.

Proof of Theorem 2.2 Given a concave, u.s.c. (w.r. to $\|\cdot\|_{\infty}$ ) function $U: \mathbb{L}^{\infty}(\Omega, \mathcal{F}, \mathbf{P}) \rightarrow \mathbb{R} \cup\{-\infty\}$ we may define the conjugate

$$
\begin{aligned}
V: \mathbb{L}^{\infty}(\Omega, \mathcal{F}, \mathbf{P})^{*} & \rightarrow \mathbb{R} \cup\{+\infty\} \\
V(\mu) & =\sup _{X \in \mathbb{L}^{\infty}}\{U(X)-\langle\mu, X\rangle\}, \quad \mu \in\left(\mathbb{L}^{\infty}\right)^{*} .
\end{aligned}
$$

We know from the general theory [R 97] and [ET 74] that $V$ is convex and l.s.c. with respect to $\sigma\left(\left(\mathbb{L}^{\infty}\right)^{*}, \mathbb{L}^{\infty}\right)$ and

$$
U(X)=\inf _{\mu \in\left(\mathbb{L}^{\infty}\right)^{*}}\{V(\mu)+\langle\mu, X\rangle\}, \quad X \in \mathbb{L}^{\infty} .
$$


If $U$ is transformation invariant then $V$ is so too. Admitting the above Proposition 4.1 we conclude that $V$ equals the $\sigma^{*}$-lower semi-continuous extension of its restriction to $\mathbb{L}^{1}$, so that

$$
U(X)=\inf _{Y \in \mathbb{L}^{1}}\{V(Y)+\mathbf{E}[Y X]\}, \quad X \in \mathbb{L}^{\infty} .
$$

Hence $\mathrm{U}$ is $\sigma^{*}$ upper semi-continuous as it is the infimum of a family of $\sigma^{*}$ continuous functions.

To prepare the proof of Proposition 4.1 we need some auxiliary results.

Lemma 4.2 For $p \in[1, \infty]$, Let $D$ be a convex, $\|\cdot\|_{p}$-closed, law invariant subset of $\mathbb{L}^{p}(\Omega, \mathcal{F}, \mathbf{P})$, and let $X \in D$. Then, for any sub-sigma-algebra $\mathcal{G}$ of $\mathcal{F}$, we have $\mathbf{E}[X \mid \mathcal{G}] \in D$.

We provide two somewhat alternative arguments for the cases $p=\infty$ and $p<\infty$.

Proof of Lemma $4.2(p=\infty)$

Step 1: We first suppose that $\mathcal{G}$ is trivial so that $\mathbf{E}[X \mid \mathcal{G}]=\mathbf{E}[X]$.

Given $X \in \mathbb{L}^{\infty}$ and $\varepsilon>0$, we may find natural numbers $M \leq N$ and a partition $A_{1}, \ldots, A_{N}$ of $\Omega$ into $\mathcal{F}$-measurable sets of probability $N^{-1}$, such that

(i) $\operatorname{osc}\left\{X \mid A_{i}\right\}<\varepsilon$, for $i=M+1, \ldots, N$,

(ii) $M / N<\varepsilon$.

Here osc $\left\{X \mid A_{i}\right\}$ denotes the essential oscillation of $X$ on $A_{i}$, i.e. the difference of the essential sup and the essential inf of $X$ on $A_{i}$.

For $1 \leq i<j \leq N$ fix a measure-preserving map $\tau_{i, j}: A_{i} \rightarrow A_{j}$ and let $\tau_{j, i}=\tau_{i, j}^{-1}$. For $\tau_{i, i}$ we choose the identity on $A_{i}$.

For a permutation $\pi:\{1, \ldots, N\} \rightarrow\{1, \ldots, N\}$ we denote by $\tau^{\pi}: \Omega \rightarrow \Omega$ the measure preserving transformation defined via $\left.\tau^{\pi}\right|_{A_{i}}=\tau_{i, \pi(i)}$.

Denoting by $\Pi_{N}$ the set of permutations of $\{1, \ldots, N\}$, the element

$$
\bar{X}:=\frac{1}{N !} \sum_{\pi \in \Pi_{N}} X \circ \tau^{\pi}
$$

is in $D$, as $D$ is convex and transformation invariant (Lemma A.4). An elementary estimate yields

$$
\|\bar{X}-\mathbf{E}[X]\|_{\infty} \leq \varepsilon+\frac{M}{N} \operatorname{osc}(X) .
$$

As $D$ is $\|\cdot\|_{\infty}$-closed we infer that $\mathbf{E}[X]$ is in $D$ too.

Step 2: Now suppose that $\mathcal{G}$ is finite, hence generated by a partition $\left.\overline{\left\{B_{1}, \ldots\right.}, B_{n}\right\}$ of $\Omega$ with $\mathbf{P}\left[B_{j}\right]>0$. 
In this case it suffices to apply step 1 on each atom $B_{j}$ to obtain the conclusion of the lemma.

Step 3: For a general sub-sigma-algebra $\mathcal{G}$ of $\mathcal{F}$ and given $X \in \mathbb{L}^{\infty}$, we may

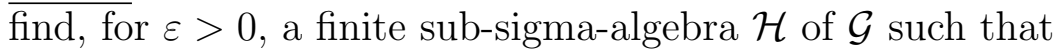

$$
\|\mathbf{E}[X \mid \mathcal{G}]-\mathbf{E}[X \mid \mathcal{H}]\|_{\infty}<\varepsilon
$$

Hence the general case follows from step 2.

Proof of Lemma $4.2(p \in[1, \infty[)$ Assume to the contrary that $\mathbf{E}[X \mid \mathcal{G}]$ does not lie in the $\|\cdot\|_{p}$-closed convex hull of the set $D$. Then, it follows from the Hahn-Banach separation Theorem that

$$
\mathbf{E}\{Z \mathbf{E}[X \mid \mathcal{G}]\}>\sup _{Y \in D} \mathbf{E}[Z Y] \quad \text { for some } Z \in \mathbb{L}^{q}(\Omega, \mathcal{F}, \mathbf{P})
$$

where $p^{-1}+q^{-1}=1$. Let $F_{Z \mid \mathcal{G}}(x):=\mathbf{P}[Z \leq x \mid \mathcal{G}]$ be the $\mathcal{G}$-conditional cumulative distribution function of $Z$, and let $q_{Z \mid \mathcal{G}}(\alpha):=\inf \left\{x \mid F_{Z \mid \mathcal{G}}(x) \geq \alpha\right\}$ be its inverse. Let $\nu$ be a random variable on $(\Omega, \mathcal{F}, \mathbf{P})$ with uniform distribution on $(0,1)$ conditionally on $\mathcal{G}$, and define

$$
\nu_{Z}:=F_{Z \mid \mathcal{G}}(Z) \mathbf{1}_{\left\{\Delta F_{Z \mid \mathcal{G}}(Z)=0\right\}}+\left(F_{Z \mid \mathcal{G}}(Z-)+\nu \Delta F_{Z \mid \mathcal{G}}(Z)\right) \mathbf{1}_{\left\{\Delta F_{Z \mid \mathcal{G}}(Z)>0\right\}},
$$

so that $Z=q_{Z \mid \mathcal{G}}\left(\nu_{Z}\right)$ a.s. Next, set $\widehat{X}:=q_{X \mid \mathcal{G}}\left(\nu_{Z}\right)$, and observe that $\widehat{X}$ has the same $\mathcal{G}$-conditional distribution as $X$ (in particular $\widehat{X} \in D$ ), and $\widehat{X}$ is comonotone to $Z$ conditionally to $\mathcal{G}$, see [JN 04]. It then follows from (19) that

$$
\mathbf{E}\{Z \mathbf{E}[X \mid \mathcal{G}]\}>\mathbf{E}[Z \widehat{X}]=\mathbf{E}\{\mathbf{E}[Z \widehat{X} \mid \mathcal{G}]\}
$$

From the $\mathcal{G}$-conditional comonotonicity of $\widehat{X}$ and $Z$, we have $\mathbf{E}[Z \widehat{X} \mid \mathcal{G}] \geq$ $\mathbf{E}[Z \mid \mathcal{G}] \mathbf{E}[\widehat{X} \mid \mathcal{G}]$, see Proposition 4 in [JN 04]. Recalling that $\widehat{X}$ and $X$ have the same $\mathcal{G}$-conditional distribution, this provides $\mathbf{E}\{Z \mathbf{E}[X \mid \mathcal{G}]\}>$ $\mathbf{E}\{\mathbf{E}[Z \mid \mathcal{G}] \mathbf{E}[\widehat{X} \mid \mathcal{G}]\}=\mathbf{E}\{Z \mathbf{E}[X \mid \mathcal{G}]\}$, which is the required contradiction.

For $\mu \in \mathbb{L}^{\infty}(\Omega, \mathcal{F}, \mathbf{P})^{*}$ and a sub- $\sigma$-algebra $\mathcal{G}$ of $\mathcal{F}$, we define the conditional expectation

$$
\mu \in \mathbb{L}^{\infty}(\Omega, \mathcal{F}, \mathbf{P})^{*} \longmapsto \mathbf{E}[\mu \mid \mathcal{G}] \in \mathbb{L}^{\infty}(\Omega, \mathcal{F}, \mathbf{P})^{*}
$$

as the transpose of the $\mathcal{G}$-conditional expectation operator on $\mathbb{L}^{\infty}(\Omega, \mathcal{F}, \mathbf{P})$, i.e.

$$
\langle\mathbf{E}[\mu \mid \mathcal{G}], \xi\rangle=\langle\mu, \mathbf{E}[\xi \mid \mathcal{G}]\rangle \text { for } \mu \in \mathbb{L}^{\infty}(\Omega, \mathcal{F}, \mathbf{P})^{*} \text { and } \xi \in \mathbb{L}^{\infty}(\Omega, \mathcal{F}, \mathbf{P})
$$

If the singular part of $\mu$ is zero, i.e. $\mu$ is absolutely continuous with respect to $\mathbf{P}$ with density $d \mu / d \mathbf{P}=Z$, then it is immediately checked that this definition coincides with the classical notion of conditional expectation in the sense that $\mathbf{E}[\mu \mid \mathcal{G}]=\mathbf{E}[Z \mid \mathcal{G}] \cdot \mathbf{P}$. 
Lemma 4.3 If $C$ is a $\sigma^{*}$-closed, convex law invariant subset of $\mathbb{L}^{\infty}(\Omega, \mathcal{F}, \mathbf{P})^{*}$, $\mu \in C$, and $\mathcal{G}$ is a sub-sigma-algebra of $\mathcal{F}$, then $\mathbf{E}[\mu \mid \mathcal{G}] \in C$.

Hence $C \cap \mathbb{L}^{1}$ is $\sigma^{*}$-dense in $C$.

Proof Fix $\mu \in C$ and the sigma-algebra $\mathcal{G}$, and suppose that $\mathbf{E}[\mu \mid \mathcal{G}] \notin C$. By the Hahn-Banach theorem there is $X \in \mathbb{L}^{\infty}$ such that

$$
a:=\sup _{\nu \in C}\langle X, \nu\rangle<\langle X, \mathbf{E}[\mu \mid \mathcal{G}]\rangle=: b .
$$

Let $D_{X}$ denote the $\|\cdot\|_{\infty}$-closed convex hull of the set $\{X \circ \tau$ । $\tau$ measure preserving transformation of $\Omega\}$. As $C$ is law invariant and therefore transformation invariant by Lemma A.5, we get

$$
a=\sup _{\nu \in C, Z \in D_{X}}\langle Z, \nu\rangle
$$

By the previous Lemma 4.2 we conclude that

$$
a \geq \sup _{\nu \in C}\langle\mathbf{E}[X \mid \mathcal{G}], \nu\rangle \geq\langle\mathbf{E}[X \mid \mathcal{G}], \mu\rangle
$$

Whence

$$
\langle\mathbf{E}[X \mid \mathcal{G}], \mu\rangle \leq a<b=\langle X, \mathbf{E}[\mu \mid \mathcal{G}]\rangle=\langle\mathbf{E}[X \mid \mathcal{G}], \mu\rangle,
$$

a contradiction proving the first assertion of the lemma.

As regards the final assertion, note that the net $\mathbf{E}[\mu \mid \mathcal{G}]$, when $\mathcal{G}$ runs through the finite sub-sigma-algebras of $\mathcal{F}$ converges to $\mu$ with respect to $\sigma\left(\left(\mathbb{L}^{\infty}\right)^{*}, \mathbb{L}^{\infty}\right)$.

Proof of Proposition 4.1 The first assertion is proved in Lemma 4.3, and the second one follows by applying it to the level sets $\{V \leq c\}$, for $c \in \mathbb{R}$.

Remark 4.4 Proposition 4.1 can be re-phrased as follows: an $\|\cdot\|_{\infty}$-closed, convex, law invariant subset $C \subseteq \mathbb{L}^{\infty}(\Omega, \mathcal{F}, \mathbf{P})$ is $\sigma\left(\mathbb{L}^{\infty}, \mathbb{L}^{1}\right)$-closed. Indeed, consider the polar $C^{\circ}=\left\{\mu \in\left(\mathbb{L}^{\infty}\right)^{*} \mid\langle\mu, f\rangle \leq 1\right.$ for $\left.f \in C\right\}$, which is a $\sigma\left(\left(\mathbb{L}^{\infty}\right)^{*}, \mathbb{L}^{\infty}\right)$-closed, convex, law invariant subset of $\left(\mathbb{L}^{\infty}\right)^{*}$. The assertion that $C^{\circ} \cap \mathbb{L}^{1}$ is $\sigma\left(\left(\mathbb{L}^{\infty}\right)^{*}, \mathbb{L}^{\infty}\right)$-dense in $C^{\circ}$ is tantamount to the $\sigma\left(\mathbb{L}^{\infty}, \mathbb{L}^{1}\right)$ closedness of $C$.

\section{The Lebesgue property for law invariant utility functions}

In this section, we provide a proof of Theorem 2.4. We first state (without proof) an easy result from measure theory which will be used in the implication (ii) $\Longrightarrow$ (i) below. 
Lemma 5.1 Let $C$ be a uniformly integrable subset of $\mathbb{L}^{1}(\Omega, \mathcal{F}, \mathbf{P})$ and $\left(X_{n}\right)_{n=1}^{\infty}$ a uniformly bounded sequence in $\mathbb{L}^{\infty}(\Omega, \mathcal{F}, \mathbf{P})$ tending a.s. to $X$. Then

$$
\lim _{n \rightarrow \infty} \inf _{Y \in C} \mathbf{E}\left[X_{n} Y\right]=\inf _{Y \in C} \mathbf{E}[X Y]
$$

\section{Proof of Theorem 2.4}

(i) $\Rightarrow$ (ii): As the Lebesgue property implies the Fatou property we know from the general theory ([D 02] and [FS 04]) that the function

$$
V(Y)=\sup _{X \in \mathbb{L}^{\infty}}\{U(X)-\mathbf{E}[X Y]\}, \quad Y \in \mathbb{L}^{1},
$$

defines the conjugate function of $U$, for which we then have the dual relation

$$
U(X)=\inf _{Y \in \mathbb{L}^{1}}\{V(Y)+\mathbf{E}[X Y]\}, \quad X \in \mathbb{L}^{\infty} .
$$

Clearly $V$ is lower semi-continuous and $V$ is law invariant iff $U$ is so.

We have to show that $\{V \leq c\}$ is uniformly integrable, for each $c>0$. Suppose to the contrary that for some $c>0$ the set $\{V \leq c\}$ fails to be uniformly integrable. Then there exists $\alpha>0$, a sequence $\left(Y_{n}\right)_{n=1}^{\infty}$ in $\{V \leq c\}$, and a sequence $\left(A_{n}\right)_{n=1}^{\infty}$ in $\mathcal{F}$, such that $\lim _{n \rightarrow \infty} \mathbf{1}_{A_{n}}=0$ a.s. and

$$
\mathbf{E}\left[\mathbf{1}_{A_{n}} Y_{n}\right] \geq \alpha>0
$$

For $X_{n}=-\frac{2 c}{\alpha} \mathbf{1}_{A_{n}}$ we find

$$
\begin{aligned}
U\left(X_{n}\right) & \leq V\left(Y_{n}\right)+\mathbf{E}\left[X_{n} Y_{n}\right] \\
& \leq c-\alpha \frac{2 c}{\alpha}=-c
\end{aligned}
$$

while $U\left(\lim _{n \rightarrow \infty} X_{n}\right)=U(0)=0$, a contradiction to the Lebesgue property of $U$.

(ii) $\Rightarrow$ (i): For given $V: \mathbb{L}^{1} \rightarrow[0, \infty]$, we know from the general theory ([D 02] and [FS 04]) that $U$ as defined in (4) is a monetary utility function satisfying the Fatou property, i.e., for uniformly bounded $\left(X_{n}\right)_{n=1}^{\infty}$ tending a.s. to $X$ we have

$$
U(X) \geq \lim _{n \rightarrow \infty} U\left(X_{n}\right)
$$

where we may assume w.l.g. that the limit on the right hand side exists. Hence we only have to show the reverse inequality of $(25)$.

So let $\left(X_{n}\right)_{n=1}^{\infty}$ be as above such that $\left(U\left(X_{n}\right)\right)_{n=1}^{\infty}$ converges. As $U$ is a monetary utility function and $\left(X_{n}\right)_{n=1}^{\infty}$ is uniformly bounded, this limit is finite.

For $\varepsilon>0$ and $n \in \mathbb{N}$ we may use (4) to find $c_{n} \geq 0$ such that

$$
U\left(X_{n}\right) \geq \inf _{V(Y) \leq c_{n}} \mathbf{E}\left[X_{n} Y\right]+c_{n}-\varepsilon
$$


Using again the fact that $\left(X_{n}\right)_{n=1}^{\infty}$ is uniformly bounded we conclude that $\left(c_{n}\right)_{n=1}^{\infty}$ is bounded, so that we may find a cluster point $c \geq 0$ and there is an infinite subset $I$ of $\mathbb{N}$ such that for $n \in I$ we have $\left|c_{n}-c\right|<\varepsilon$. We then may apply the preceding Lemma 5.1 to estimate

$$
\begin{aligned}
U(X) & \leq \inf _{V(Y) \leq c+\varepsilon} \mathbf{E}[X Y]+(c+\varepsilon) \\
& =\lim _{n \in I} \inf _{V(Y) \leq c+\varepsilon} \mathbf{E}\left[X_{n} Y\right]+c+\varepsilon \\
& \leq \lim _{n \in I} \inf _{V(Y) \leq c_{n}} \mathbf{E}\left[X_{n} Y\right]+c+\varepsilon \\
& \leq \lim _{n \rightarrow \infty} U\left(X_{n}\right)+3 \varepsilon .
\end{aligned}
$$

(ii) $\Longleftrightarrow$ (iii) In view of the first step of the proof of Theorem 2.1 (ii) $\Leftrightarrow$ (iii) in Section 3, it only remains to show that the notion of uniform integrability in (ii) corresponds to the notion of relative compactness with respect to the Prokhorov topology in (iii). To do so, note that if $m \in \mathcal{P}([0,1])$ and $f \in \mathcal{D} \searrow$ are related via $T(f)=m$, see (6), we have for $\alpha \in] 0,1]$ (arguing once more by approximation with bounded, differentiable functions $f$ )

$$
\begin{aligned}
m(] 0, \alpha[) & =\int_{0}^{\alpha} d m(x) \\
& =-\int_{0}^{\alpha} x f^{\prime}(x) d x \\
& =-[x f(x)]_{0}^{\alpha}+\int_{0}^{\alpha} f(x) d x \\
& =\int_{0}^{\alpha}(f(x)-f(\alpha)) d x .
\end{aligned}
$$

The set $\left\{Y \in \mathbb{L}^{1} \mid V(Y) \leq c\right\}$ is uniformly integrable iff the integral $\int_{0}^{\alpha}(f(x)-$ $f(\alpha)) d x$ tends to zero, for $\alpha \rightarrow 0$, uniformly over the set $\left\{f=-q_{-Y} \in \mathcal{D} \backslash \mid\right.$ $V(Y) \leq c\}$. In view of $(28)$ this is tantamount to the fact that $m(] 0, \alpha[)$ tends to zero, for $\alpha \rightarrow 0$, uniformly over the set $\{m \in \mathcal{P}(] 0,1]) \mid v(m) \leq c\}$.

In the above proof of the equivalence of (i) and (ii) of Theorem 2.4 we have not used the law invariance of $U$ and $V$ respectively, which is irrelevant for this equivalence (while for the formulation of (iii) it is, of course, indispensable).

In fact this is part of a more general characterization of the Lebesgue property in equivalent terms as mentioned in the beginning of this section. As these results are somewhat scattered in the previous literature [D 03], [FS 04], [K 05] we resume them in the subsequent theorem and give proofs. In the subsequent theorem, we identify $\mathbb{L}^{1}(\Omega, \mathcal{F}, \mathbf{P})$ with a subspace of $\mathbb{L}^{\infty}(\Omega, \mathcal{F}, \mathbf{P})^{*}$.

Theorem 5.2 Suppose that $\mathbb{L}^{1}(\Omega, \mathcal{F}, \mathbf{P})$ is separable, let $U: \mathbb{L}^{\infty}(\Omega, \mathcal{F}, \mathbf{P}) \rightarrow$ $\mathbb{R}$ be a monetary utility function satisfying the Fatou property and let 
$V: \mathbb{L}^{\infty}(\Omega, \mathcal{F}, \mathbf{P})^{*} \rightarrow[0, \infty]$ be its conjugate function defined on the dual $\mathbb{L}^{\infty}(\Omega, \mathcal{F}, \mathbf{P})^{*}$, i.e.

$$
V(\mu):=\sup _{X \in \mathbb{L}^{\infty}}\{U(X)-\langle\mu, X\rangle\}, \quad \mu \in \mathbb{L}^{\infty}(\Omega, \mathcal{F}, \mathbf{P})^{*} .
$$

The following assertions are equivalent:

(i) U satisfies the Lebesgue property.

(ii) $U(x)$ is continuous from below in the sense of [FS04], i.e. for every sequence $\left(X_{n}\right)_{n=1}^{\infty} \in \mathbb{L}^{\infty}$ increasing monotonically to $X \in \mathbb{L}^{\infty}$ we have $\lim _{n \rightarrow \infty} U\left(X_{n}\right)=U(X)$.

(iii) $\operatorname{dom}(V)=\{V<\infty\} \subseteq \mathbb{L}^{1}(\Omega, \mathcal{F}, \mathbf{P})$.

(iv) For each $c \in \mathbb{R},\{V \leq c\}$ is

(v) For every $X \in \mathbb{L}^{\infty}(\Omega, \mathcal{F}, \mathbf{P})$ the infimum in the equality

$$
U(X)=\inf _{Y \in \mathbb{L}^{1}}\{V(Y)+\mathbf{E}[X Y]\}
$$

is attained.

Remark 5.3 In the above theorem, the only requirement on the probability space is that $\mathbb{L}^{1}(\Omega, \mathcal{F}, \mathbf{P})$ is separable (we need this assumption for the implication $(\mathrm{v}) \Rightarrow(\mathrm{iv}))$. In particular, $(\Omega, \mathcal{F}, \mathbf{P})$ need not be atomless.

Remark 5.4 The notion of continuity from below was introduced in [FS 04, Proposition 4.21], where the equivalence of (ii) and (iii) was shown. Property (v) was studied in [D 03] where, applying James' theorem, F. Delbaen showed the equivalence of (iv) and (v) in the context of coherent risk measures. After finishing the paper we were kindly informed by F. Delbaen that he has an argument to directly deduce the above implication $(\mathrm{v}) \Rightarrow$ (iv) from James' theorem also for the case of convex risk measures without referring to a variant of this theorem such as Theorem A.1 below [D 05].

\section{Proof of Theorem 5.2}

(i) $\Leftrightarrow($ iv): We have shown this equivalence in the proof of Theorem 2.4 above. (iii) $\Leftrightarrow($ iv): the implication (iv) $\Rightarrow$ (iii) being obvious note for the converse that a subset $C \subseteq \mathbb{L}^{1}(\Omega, \mathcal{F}, \mathbf{P})$ is relatively weakly compact iff its $\sigma\left(\left(\mathbb{L}^{\infty}\right)^{*}, \mathbb{L}^{\infty}\right)$ closure is contained in $\mathbb{L}^{1}(\Omega, \mathcal{F}, \mathbf{P})$. As the level sets $\left\{\mu \in\left(\mathbb{L}^{\infty}\right)^{*} \mid V(\mu) \leq c\right\}$ are the $\sigma\left(\left(\mathbb{L}^{\infty}\right)^{*}, \mathbb{L}^{\infty}\right)$ closure of $\left\{Y \in \mathbb{L}^{1} \mid V(Y) \leq c\right\}$ in view of the Fatou property of $U$, we obtain (iii) $\Rightarrow$ (iv).

(i) $\Rightarrow$ (ii) obvious.

(ii) $\Rightarrow$ (iv): This implication was shown in (i) $\Rightarrow$ (ii) of Theorem 2.4.

(iv) $\Rightarrow(\mathrm{v})$ : For $X \in \mathbb{L}^{\infty}(\Omega, \mathcal{F}, \mathbf{P})$ the function

$$
c \mapsto \inf _{V(Y) \leq c} \mathbf{E}[X Y]
$$


is decreasing, convex and bounded on $[0, \infty[$ so that the function

$$
c \mapsto \inf _{V(Y) \leq c} \mathbf{E}[X Y]+c
$$

attains its minimum at some $\bar{c} \in[0, \infty[$. Hence

$$
U(X)=\inf _{V(Y) \leq \bar{c}} \mathbf{E}[X Y]+\bar{c}
$$

As $\{V(Y) \leq \bar{c}\}$ is a weakly compact set in $\mathbb{L}^{1}(\Omega, \mathcal{F}, \mathbf{P})$ the infimum in (30) is attained.

(v) $\Rightarrow$ (iv) To prove this implication first suppose that $U$ is positively homogenous, i.e. $\rho=-U$ is a coherent risk measure. In this case $\{V<\infty\}=\{V=$ $0\}$.

If $\left\{Y \in \mathbb{L}^{1} \mid V(Y)=0\right\}$ fails to be weakly compact, then we deduce from James' theorem (see, e.g., [FLP 01], [FHMPZ 01]) that there is some $X \in$ $\mathbb{L}^{\infty}(\Omega, \mathcal{F}, \mathbf{P})$ such that $X$ does not attain its infimum on $\left\{Y \in \mathbb{L}^{1} \mid V(Y)=0\right\}$. Hence in the equation

$$
U(X)=\inf _{Y \in \mathbb{L}^{1}, V(Y)=0} \mathbf{E}[X Y]
$$

the infimum is not attained.

Now we drop the assumption that $U$ is positively homogenous. In this case one needs to apply a variant of James' theorem, which was shown to us by P. Orihuela — following closely the arguments of [G 87] — and which we state and prove in the appendix A.1. This theorem implies that, whenever (iv) fails, we may find $X \in \mathbb{L}^{\infty}(\Omega, \mathcal{F}, \mathbf{P})$ such that in

$$
U(X)=\inf _{Y \in \mathbb{L}^{1}}\{\mathbf{E}[X Y]+V(Y)\}
$$

the infimum is not attained.

We still note that we may rephrase condition (iii) of Theorem 2.1 again in terms of the set $\mathcal{D} \searrow$ (similarly as in (14) above for the case of Theorem 2.1):

(iii') There is a convex function $\mathcal{V}: \mathcal{D} \backslash \rightarrow[0,1]$ such that

$$
U(X)=\inf _{f \in \mathcal{D} \backslash}\left\{\int_{0}^{1} q_{X}(\alpha) f(\alpha) d \alpha-\mathcal{V}(f)\right\} .
$$

The verification that this condition is indeed equivalent to condition (ii) and (iii) in Theorem 2.1 is similar as for (14) above. 


\section{A Appendix}

The proof of the variant of James' theorem below was communicated to us by P. Orihuela. We sincerely thank him for allowing us to include it in this paper.

Theorem A.1 Let $(E,\|\|$.$) be a separable Banach space and V: E \rightarrow \mathbb{R} \cup$ $\{\infty\}$ a proper convex l.s.c. function such that $\operatorname{dom}(V)=\{V<\infty\}$ is a bounded subset of $E$. Suppose that there is $c \in \mathbb{R}$ such that the level set $L_{c}=\{V \leq c\}$ fails to be weakly compact.

Then there is $x^{*} \in E^{*}$ such that, for

$$
U\left(x^{*}\right)=\inf _{x \in E}\left\{\left\langle x, x^{*}\right\rangle+V(x)\right\}
$$

the infimum is not attained.

To prove the theorem we recall the inequality of Simons which isolates the combinatorial part in James' theorem.

Proposition A.2 ([S 72], [G 87]): Let $B$ be a set and $\left(f_{n}\right)_{n=1}^{\infty}$ a sequence of functions on $B$ taking their values in a compact interval $[a, b]$. Denote by $C$ the convex set

$$
C=\left\{\sum_{n=1}^{\infty} c_{n} f_{n} \mid c_{n} \geq 0, \sum_{n=1}^{\infty} c_{n}=1\right\},
$$

and suppose that every element $f \in C$ attains its infimum on $B$. Then

$$
\inf _{b \in B} \liminf _{n \rightarrow \infty} f_{n}(b) \leq \sup _{f \in C} \inf _{b \in B} f(b) .
$$

We also need an elementary estimate.

Lemma A.3 Let $V: E \rightarrow \mathbb{R} \cup\{\infty\}$ be as in Theorem A.1; suppose that $\operatorname{dom}(V)$ is contained in the unit ball of $E$ and that $\inf _{x \in E} V(x)<0$.

Denoting by

$$
\begin{aligned}
\operatorname{Epi}(V) & =\{(x, t) \in E \times \mathbb{R} \mid V(x) \leq t\}, \\
\text { and } \quad \operatorname{Epi}(V, \mu) & =\{(x, t) \in E \times \mathbb{R} \mid V(x) \leq t \leq \mu\}, \quad \mu \in \mathbb{R},
\end{aligned}
$$

let $\left(x^{*}, \lambda\right) \in E^{*} \times \mathbb{R},\left\|x^{*}\right\| \leq 1, \lambda>0$.

Then, for $\mu \geq 2 \lambda^{-1}$, we have

$$
\inf _{(x, t) \in \operatorname{Epi}(V)}\left\langle\left(x^{*}, \lambda\right),(x, t)\right\rangle=\inf _{(x, t) \in \operatorname{Epi}(V, \mu)}\left\langle\left(x^{*}, \lambda\right),(x, t)\right\rangle .
$$

Proof Fix $x_{0} \in X,\left\|x_{0}\right\| \leq 1$, such that $V\left(x_{0}\right) \leq 0$. Then, for every $(x, t) \in$ $\operatorname{Epi}(V)$ such that

$$
\left\langle\left(x^{*}, \lambda\right),(x, t)\right\rangle \leq\left\langle\left(x^{*}, \lambda\right),\left(x_{0}, 0\right)\right\rangle
$$


we have

$$
\lambda t \leq\left\langle x^{*}, x_{0}-x\right\rangle \leq 2 .
$$

Proof of Theorem A.1 Assume w.l.g. that $\operatorname{dom}(V)$ is contained in the unit ball of $E$ and that $\inf _{x \in E} V(x)=-1$. Assume that for every $x^{*} \in E^{*}$ the infimum in

$$
U\left(x^{*}\right):=\inf _{x \in E}\left\{\left\langle x, x^{*}\right\rangle+V(x)\right\}
$$

is attained and let us work towards a contradiction.

Consider the Banach space $E^{* *} \times \mathbb{R}$, in which the epigraph (33) of $V$, is a norm-closed convex set.

Note that the optimisation problem (35) may be rewritten as

$$
U\left(x^{*}\right)=\inf _{(x, t) \in \operatorname{Epi}(V)}\left\{\left\langle\left(x^{*}, 1\right),(x, t)\right\rangle\right\},
$$

and that, for $x^{*} \in E^{*}$, the inf in (35) is attained iff the inf in (36) is attained. Hence the inf in (35) is attained for each $x^{*} \in E^{*}$ iff each $\left(x^{*}, \lambda\right)$, where $x^{*} \in E^{*}$ and $\lambda>0$, attains its inf on $\operatorname{Epi}(V)$.

By hypothesis there is some level $c>0$ such that $L_{c}=\{V \leq c\}$ is not weakly compact in $E$.

By the convexity of $V$ this holds true for every $c>\inf _{x \in E} V(x)=-1$ so that we may choose $c=0$.

Hence there is $\left(x^{* *}, 0\right) \in\left(E^{* *} \times \mathbb{R}\right) \backslash(E \times \mathbb{R})$ which is in the $\sigma\left(E^{* *} \times \mathbb{R}, E^{*} \times\right.$ $\mathbb{R})$-closure of $\operatorname{Epi}(V)$. We may apply Hahn-Banach to separate $\operatorname{Epi}(V)$ strictly from $\left(x^{* *}, 0\right)$, i.e. there are $\left(x^{* * *}, \lambda\right) \in E^{* * *} \times \mathbb{R}$ with $\left\|x^{* * *}\right\| \leq 1$ and $\alpha<\beta$ such that

$$
\left\langle\left(x^{* * *}, \lambda\right),\left(x^{* *}, 0\right)\right\rangle<\alpha<\beta<\inf _{(x, t) \in \operatorname{Epi}(V)}\left\langle\left(x^{* * *}, \lambda\right),(x, t)\right\rangle .
$$

Clearly we must have $\lambda \geq 0$. In fact, we may assume that $\lambda>0$. Indeed if (37) holds true for some $\left(x^{* * *}, \lambda\right)$ then it also holds true for $\left(x^{* * *}, \lambda^{\prime}\right)$ provided that $\lambda \leq \lambda^{\prime}<\inf _{(x, t) \in \operatorname{Epi}(V)}\left\langle\left(x^{* * *}, \lambda\right),(x, t)\right\rangle-\beta$. Indeed, the passage from $\lambda$ to $\lambda^{\prime}$ does not affect the first inequality of (37) while the last one remains valid in view of $t \geq-1$, for $(x, t) \in \operatorname{Epi}(V)$.

Fix a dense sequence $\left(x_{j}\right)_{j=1}^{\infty}$ in $E$ and use the $\sigma\left(E^{* * *}, E^{* *}\right)$-densitiy of the unit ball of $E^{*}$ in the unit ball of $E^{* * *}$ to find a sequence $\left(x_{n}^{*}\right)_{n=1}^{\infty}$ with $\left\|x_{n}^{*}\right\| \leq 1$ such that

$$
\left|\left\langle x_{n}^{*}-x^{* * *}, x_{j}\right\rangle\right|<n^{-1}, \quad j=1, \ldots, n,
$$

and $\left\langle x_{n}^{*}, x^{* *}\right\rangle<\alpha$.

By hypothesis each $\left(x_{n}^{*}, \lambda\right)$ as well as any countable convex combination of this sequence attains its inf at some $(x, \mu) \in \operatorname{Epi}(V)$ for which we find $\mu \leq 2 \lambda^{-1}$ 
by Lemma A.3. Let $\mu_{0}=2 \lambda^{-1}$ and define $B$ as the truncated epigraph (34) of $V$ at level $\mu_{0}$, i.e.

$$
B=\operatorname{Epi}\left(V, \mu_{0}\right)=\left\{(x, t) \in E \times \mathbb{R} \mid V(x) \leq t \leq \mu_{0}\right\},
$$

which is a bounded subset of $E \times \mathbb{R}$.

We now are in a position to apply Simons' inequality. Letting $C=$ $\left\{\sum_{n=1}^{\infty} c_{n}\left(x_{n}^{*}, \lambda\right) \mid c_{n} \geq 0, \sum_{n=1}^{\infty} c_{n}=1\right\}$ we have

$$
a:=\sup _{\left(x^{*}, \lambda\right) \in C} \inf _{(x, t) \in B}\left\langle\left(x^{*}, \lambda\right),(x, t)\right\rangle \leq \alpha .
$$

Indeed, for every $\left(x^{*}, \lambda\right) \in C$ we have $\left\langle\left(x^{*}, \lambda\right),\left(x^{* *}, 0\right)\right\rangle \leq \alpha$; noting that $\left(x^{* *}, 0\right)$ is in the $\sigma\left(E^{* *} \times \mathbb{R}, E^{*} \times \mathbb{R}\right)$-closure of $B=\operatorname{Epi}\left(V, \mu_{0}\right)$ and $\left(x^{*}, \lambda\right)$ is continuous with respect to this topology, we obtain (38).

On the other hand

$$
b:=\inf _{(x, t) \in B} \liminf _{n \rightarrow \infty}\left\langle\left(x_{n}^{*}, \lambda\right),(x, t)\right\rangle \geq \beta .
$$

Indeed, by construction we have $\lim _{n \rightarrow \infty}\left\langle x_{n}^{*}, x\right\rangle=\left\langle x^{* * *}, x\right\rangle$, for every $x \in E$, so that (39) follows from (37). Hence (32) yields the desired contradiction

$$
b \leq a \leq \alpha<\beta \leq b .
$$

We finish the paper by two easy measure theoretic results.

Lemma A.4 Let $(\Omega, \mathcal{F}, \mathbf{P})$ be a standard probability space, $1 \leq p \leq \infty$, and $C$ a norm closed subset of $\mathbb{L}^{p}(\Omega, \mathcal{F}, \mathbf{P})$. T.f.a.e.

(i) $C$ is law invariant, i.e., $X_{1} \in C$ and law $\left(X_{1}\right)=\operatorname{law}\left(X_{2}\right)$ implies that $X_{2} \in C$.

(ii) $C$ is transformation invariant, i.e., for $X \in C$ and a bi-measurable measure preserving transformation $\tau:(\Omega, \mathcal{F}, \mathbf{P}) \rightarrow(\Omega, \mathcal{F}, \mathbf{P})$ we have $X \circ \tau \in C$.

Proof (i) $\Rightarrow$ (ii): Note that $\operatorname{law}(X)=\operatorname{law}(X \circ \tau)$ for $X$ and $\tau$ as in (ii). (ii) $\Rightarrow(\mathrm{i})$ : Let $X_{1}, X_{2} \in \mathbb{L}^{p}$ with law $\left(X_{1}\right)=\operatorname{law}\left(X_{2}\right)$. For $\varepsilon>0$ let $\left(A_{i}\right)_{i=1}^{\infty}$ be a partition of $\mathbb{R}$ into countably many sets of diameter less than $\varepsilon$; for example, one may choose the half-open intervals \{]$\left.\left.\frac{k}{2^{n}}, \frac{k+1}{2^{n}}\right]\right\}_{k \in \mathbb{Z}}$, for $n$ sufficiently large. The sets

$$
B_{i}^{1}=\left\{X_{1} \in A_{i}\right\}, B_{i}^{2}=\left\{X_{2} \in A_{i}\right\},
$$

satisfy $\mathbf{P}\left[B_{i}^{1}\right]=\mathbf{P}\left[B_{i}^{2}\right]$, for each $i \in \mathbb{N}$. Using the hypothesis that $(\Omega, \mathcal{F}, \mathbf{P})$ is a standard probability space, we may find a bi-measurable measure preserving transformation $\tau:(\Omega, \mathcal{F}, \mathbf{P}) \rightarrow(\Omega, \mathcal{F}, \mathbf{P})$ mapping each $B_{i}^{1}$ onto $B_{i}^{2}$. We then have

$$
\left\|X_{2}-X_{1} \circ \tau\right\|_{\infty} \leq \varepsilon
$$


Assumption (ii) and $X_{1} \in C$ implies that $X_{1} \circ \tau \in C$. The $\|\cdot\|_{p}$-closedness of $C$ then implies that $X_{2} \in C$.

In the next lemma we formulate an analogous result for the case of $\mathbb{L}^{\infty}(\Omega, \mathcal{F}, \mathbf{P})^{*}$.

Lemma A.5 Let $(\Omega, \mathcal{F}, \mathbf{P})$ be a standard probability space and $C$ a $\sigma^{*}$-closed, convex subset of $\mathbb{L}^{\infty}(\Omega, \mathcal{F}, \mathbf{P})^{*}$. T.f.a.e.

(i) $C$ is law invariant, i.e., for $X_{1}, X_{2} \in \mathbb{L}^{\infty}$ with $\operatorname{law}\left(X_{1}\right)=\operatorname{law}\left(X_{2}\right)$, we have

$$
\left\{\left\langle\mu, X_{1}\right\rangle \mid \mu \in C\right\}=\left\{\left\langle\mu, X_{2}\right\rangle \mid \mu \in C\right\} .
$$

(ii) $C$ is transformation invariant, i.e., $C=\tau^{*}(C)$ for each measure preserving transformation $\tau:(\Omega, \mathcal{F}, \mathbf{P}) \rightarrow(\Omega, \mathcal{F}, \mathbf{P})$ (see $(16))$.

(iii) The conjugate function $\Phi$ of $C$ defined by

$$
\Phi(X)=\sup _{\mu \in C}\langle\mu, X\rangle, \quad X \in \mathbb{L}^{\infty},
$$

is law invariant.

Proof $(\mathrm{i}) \Leftrightarrow\left(\right.$ iii): W.l.g. assume $C \neq \emptyset$. As $C$ is $\sigma^{*}$-closed and convex, for $X \in \mathbb{L}^{\infty}$ the set $I(X):=\{\langle\mu, X\rangle \mid \mu \in C\}$ is the closed non-empty interval $[-\Phi(-X), \Phi(X)]$. Obviously $C$ is law invariant iff $\Phi$ is law invariant.

(ii) $\Leftrightarrow($ iii): By Lemma A.4 the function $\Phi$ is law invariant iff it is transformation invariant, i.e., $\Phi=\Phi \circ \tau$ for each bi-measurable measure preserving $\tau:(\Omega, \mathcal{F}, \mathbf{P}) \rightarrow(\Omega, \mathcal{F}, \mathbf{P})$. Hence the equivalence of (ii) and (iii) is obvious.

\section{References}

[ADEH 97] Artzner P., Delbaen F., Eber J.M. and D. Heath (1999). Coherent measures of risk. Mathematical Finance 9, 203-228.

[D 02] Delbaen F. (2002). Coherent risk measure of risk on general probability spaces. In: Advances in Finance and Stochastics, Essays in Honor of Dieter Sondermann (K. Sandmann and P.J. Schonbucher, eds.) 1-37, Springer, Berlin.

[D 03] Delbaen F. (2003). Coherent risk measures, Lecture Notes of Scuola Normale Pisa.

[D 05] Delbaen F. (2005). private communication referring to a forthcoming paper "Coherent risk measures". 
[ET 74] Ekeland I. and R. Temam (1974). Analyse Convexe et Problèmes Variationnels, Dunod Gauthier-Villars.

[FHMPZ 01] Fabian M., P Habala, V. Montesinos, J. Pelant and V. Zizler (2001). Functional Analysis and Infinite Dimensional Geometry. Springer Verlag, New York.

[FLP 01] Fonf V.P., J. Lindenstrauss and R.R. Phelps (2001). Infinite dimensional convexity. In: Handbook of the geometry of Banach spaces. Vol. I, North-Holland-Amsterdam, 599-670.

[FG 05] Frittelli M. and E. Rossaza Gianin (2005). Law invariant convex risk measures, Advances in Mathematical Economics 7 (2005), p. 33-46.

[FS 04] Föllmer H. and A. Schied (2004). Stochastic Finance, Second Edition, de Gruyter.

[G 87] Godefroy G. (1987). Boundaries of a convex set and interpolation sets. Mathematische Annalen 277 (2), 173-184.

[JST 05] Jouini E., Schachermayer W. and N. Touzi (2005). Optimal risk sharing with law invariant monetary utility functions, preprint.

[JN 04] Jouini E. and C. Napp (2004). Conditional comonotonicity. Decisions in Economics and Finance, to appear.

[K 05] Krätschmer V. (2005). Robust representation of convex risk measures by probability measures, to appear in Finance and Stochastics.

[K 01] Kusuoka S. (2001). On law-invariant coherent risk measures. Advances in Mathematical Economics 3, 83-95.

[Ph 93] Phelps R.R. (1993). Convex functions, monotone operators and differentiability, Lecture Notes in Mathematics 1364, Springer.

[R 97] Rockafellar R.T. (1997). Convex Analysis, Princeton Landmarks in Mathematics, Princeton University Press.

[S 72] Simons S. (1972). A convergence theorem with boundary. Pacific Journal of Mathematics 40, 703-708. 